\title{
Study of Double-holding Water Tank Liquid Level Control Base on Neural Networks PID Control
}

\author{
Yuli Wei* \\ Lanzhou Resources \& Environment Voc-Tech College, Lanzhou, Gansu 730020, China \\ ${ }^{*}$ Corresponding author
}

\begin{abstract}
Aiming at the problem of the effect of longtime delay and nonlinear of the double-tank's level control, the PID control of BP neural network tuning parameters is studied in this paper. The characteristics of the control system are analyzed minutely, a neural PID controller is designed and its control algorithm is derived. Simulation control program is compiled in MATLAB, and the program is applied to the experimental platform, then a better control effect is obtained after software debugging and parameter optimization. The results show the effectiveness and correctness of the control method.
\end{abstract}

Keywords-double-holding water tank; liquid level control; BP neural networks; PID control

\section{INTRODUCTION}

As a typical representative of the process control, level control of the double-tank is one of the hotspots in the research of process control experts. Due to longtime delay, nonlinear characteristics and complexity of the control system, the control effect has been affected by the system's initial state, control algorithms and parameters directly [1-3]. The classical PID control is difficult to achieve satisfactory control effect, because the parameter optimization is not easy; Fuzzy control is a classical intelligent control method, it can achieve a better control effect as long as the design is reasonable. But fuzzy control is built on the basis of fuzzy logic reasoning, so the demands for designers' experience are stringent. of course, there are many other control methods that experts and scholars are studying $[4,5]$. Combined with the characteristics of the process control experimental platform, the application of the neural PID method is studied for the level control of the double-tank in this paper.

\section{CHARACTERISTICS OF DOUBLE-HOLDING WATER TANK}

This paper is based on the THKGK-1process control system experimental device. Combining with the upper monitor PC machine which forms a complete and complex control system, and the control system can complete the temperature, pressure, flow, liquid level control and et al, level control of the double-tank is one of them. The double-tank level control is a typical object of the process control, it has the characteristics that its own longtime delay, object's nonlinear with the parameter variation and complex of the control system, and different control algorithm affects the accuracy of control directly.
System principle is the measured object which is composed of two water tanks with different volume in series, so it is called the two-volume object. The principle of two-link water tanks is cascaded two different volume physical tanks, as shown in Figure I.

The controlled variable in this system is the liquid level of the lower water tank. When the input quantity of the upper water tank has a step increment change, the change curve of liquid level of the two water tank is shown in Figure II. Figure II (a) shows that the response curve of the upper water tank liquid level which is a monotonically increasing exponent curve. However, in Figure II (b), the response curve of the lower water tank liquid level is "S" curve. That is to say, the liquid level response of the lower tank is delayed, it has a great relationship between time delays and characteristics of the system, for instance, the opening size of water inlet valve and water outlet valve are equal.

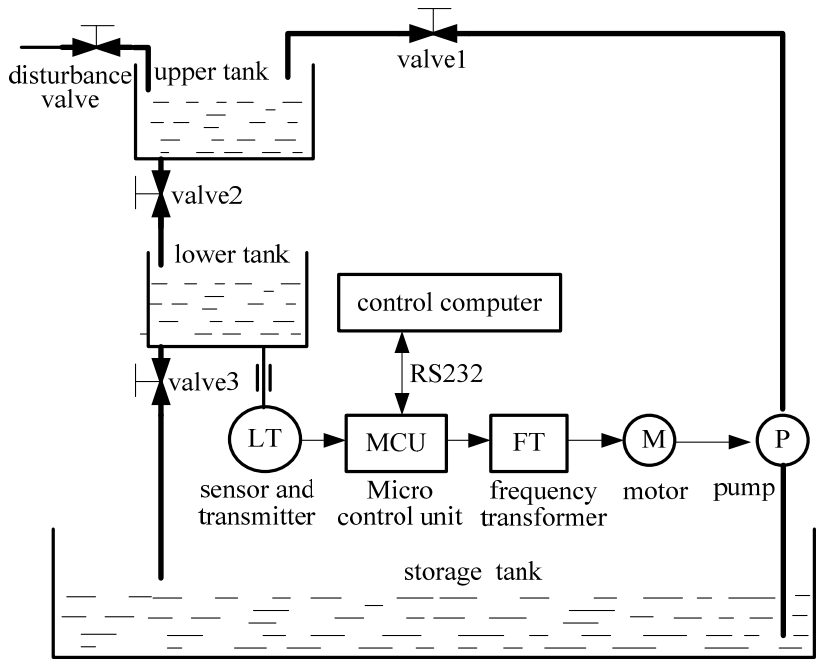

FIGURE I. THE PRINCIPLE OF TWO-LINK WATER TANKS 


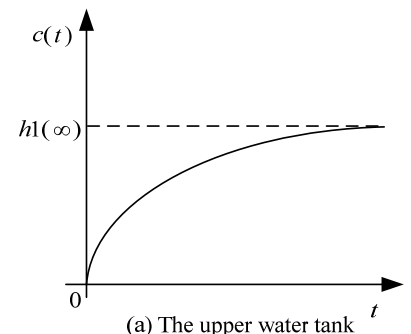

(a) The upper water tank $t$

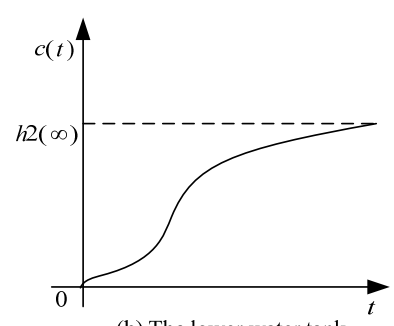

(b) The lower water tank
FIGURE II. STEP OUTPUT CURVE OF DOUBLE-TANK

The mathematical model of double-tank is the product of mathematical model of two single- container water tank, which can be described as two first-order inertial link. Coupled with system pure time delay, the transfer function can be obtained:

$$
G(s)=G_{1}(s) G_{2}(s)=\frac{k}{\left(T_{1} s+1\right)\left(T_{2} s+1\right)} e^{-\tau s}
$$

Where, $\mathrm{k}$ is the amplification factor of the double-tank, $T_{1}$ and $T_{2}$ are constant time of the double-tank, $\tau$ is the lag time.

Due to the time delays of the system is closely related to the size of the water inlet valve and water outlet valve. After changing the corresponding valve opening, the object may appear some hysterics characteristics with different degrees, so the classical control method is difficult to achieve satisfactory control effect. The fuzzy control has been used to carry on the research, the control effect is improved compared with the traditional PID control (the control algorithm of the experimental system itself), but there are still some disadvantages. For example, overshoot is too large, so this paper attempts to use the neural PID for further study.

\section{DESIGN OF NEURAL PID CONTROL}

The better effect of PID control based on the best adjustment parameter of the proportion, integral and differential, it forms the relationship of mutual coordination and mutual restraint. This relationship is not a simple linear combination, in the nonlinear combination with countless changes, it can find the best. Neural network has the ability of arbitrary nonlinear expression, which can be achieved the best combination of PID control through the study of system performance.

In this paper, BP (Propagation Back) network is used to set up the neural PID control [6, 7], a PID controller which the parameters $k_{\mathrm{p}}, k_{\mathrm{i}}, k_{\mathrm{d}}$ can study by themselves was built. In the controller, three layers network structure is adopted, the network structure and the control system structure are shown in Figure III and Figure IV.

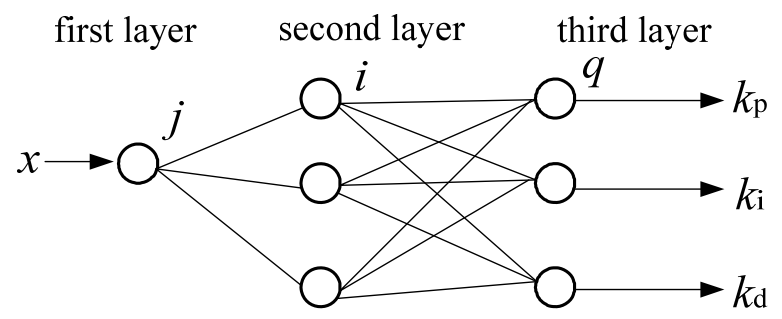

FIGURE III. NEURAL PID CONTROL SYSTEM STRUCTURE

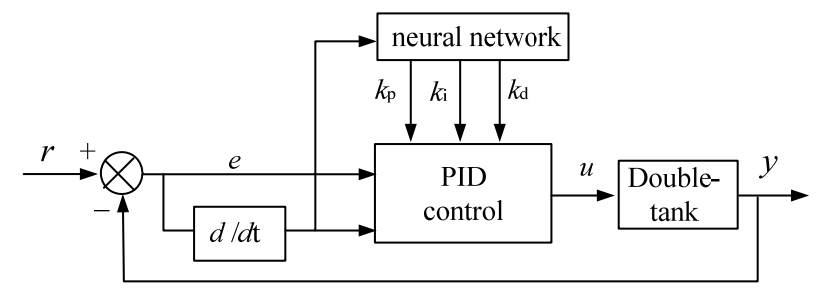

FIGURE IV. THE THREE LAYERS BP NETWORK STRUCTURE

The conventional PID control is the direct closed-loop control of the controlled device, and parameters $k_{\mathrm{p}}, k_{\mathrm{i}}, k_{\mathrm{d}}$ are offline set. Its incremental digital PID control algorithm is:

$$
\Delta u(k)=k_{\mathrm{p}}[e(k)-e(k-1)]+k_{\mathrm{i}} e(k)+k_{\mathrm{d}}[e(k)-2 e(k-1)+e(k-2)]
$$

Where, $k_{\mathrm{p}}, k_{\mathrm{i}}, k_{\mathrm{d}}$ are parameters of proportion, integral and differential respectively.

According to the running state of the system, adjusting the parameters of the PID controller can achieve the optimization of a certain performance index. The output state of the output layer neuron corresponds to the three adjustable PID controller's parameters $k_{\mathrm{p}}, k_{\mathrm{i}}, k_{\mathrm{d}}$. By the neural network learning itself and the adjustment of weighting coefficients, the output of the neural network is corresponding to the PID controller's parameters with some optimal control law. The specific network algorithm [8] is as follows:

The input of the network input layer is: $x(j)$;

The output the network input layer is: $o_{j}^{(1)}=\mathrm{x}(j), j=1$;
The input of network hidden layer is: $\operatorname{net}_{i}^{(2)}(k)=\omega_{i j}^{(2)} o_{j}^{(1)}$;

the output of network hidden layer is: $o_{j}^{(2)}(k)=f\left(n e t_{i}^{(2)}(k)\right)$, $i=1,2,3$;

The input of the network output layer is:

$$
n e t_{p}^{(3)}(k)=\sum_{i=0}^{3} \omega_{p i}^{(3)} o_{i}^{(2)} \text {; }
$$

The output is: $o_{p}^{(3)}(k)=f\left(n e t_{p}^{(3)}(k)\right), p=1,2,3$;

In the above formulas, $\omega_{i j}^{()}$is weighted coefficient; the superscript(1), (2), (3) are input layer, hidden layer and output 
layer respectively. The activation function of the hidden layer and the output layer are all the $\mathrm{S}$ function, which is $y=f(s)=1 /\left(1+e^{-n s}\right)$.

The output nodes of the output layer correspond to the three adjustable parameters, which are $o_{1}^{(3)}=k_{p}, o_{2}^{(3)}=k_{i}, o_{3}^{(3)}=k_{d}$.

Performance index function: $E(k)=\left[(r(k)-y(k)]^{2} / 2\right.$. With the improved BP network learning algorithm, the weights of the network are modified according to the negative gradient descent method:

$$
\Delta w_{p i}^{(3)}(k)=-\eta \frac{\partial E(k)}{\partial w_{p i}^{(3)}}+\alpha \Delta w_{p i}^{(3)}(k-1)
$$

Where, $\eta$ is learning rate, $\alpha$ is inertia coefficient.

$$
\begin{gathered}
\frac{\partial E(k)}{\partial w_{p i}^{3}}=\frac{\partial E(k)}{\partial y(k)} \cdot \frac{\partial y(k)}{\partial \Delta u(k)} \cdot \frac{\partial \Delta u(k)}{\partial o_{p}^{(3)}(k)} \cdot \frac{\partial o_{p}^{(3)}(k)}{\partial n e t_{p}^{(3)}} \cdot \frac{\partial n e t_{p}^{(3)}(k)}{\partial w_{p i}^{(3)}(k)}, \\
\frac{\partial n e t_{p}^{(3)}(k)}{\partial w_{p i}^{(3)}(k)}=o_{i}^{(2)}(k)
\end{gathered}
$$

According to the above analysis, the learning algorithm of the network output layer weight coefficient can be obtained:

$$
\begin{aligned}
\Delta w_{p i}^{(3)}(k) & =\alpha \Delta w_{p i}^{(3)}(k-1)-\eta \frac{\partial E(k)}{\partial y(k)} \cdot \frac{\partial y(k)}{\partial \Delta u(k)} \cdot \frac{\partial \Delta u(k)}{\partial o_{p}^{(3)}(k)} \cdot \frac{\partial o_{p}^{(3)}(k)}{\partial n e t_{p}^{(3)}} \cdot o_{i}^{(2)}(k) \\
= & \alpha \Delta w_{p i}^{(3)}(k-1)-\eta \cdot e(k) \cdot \frac{\partial y(k)}{\partial \Delta u(k)} \cdot \frac{\partial \Delta u(k)}{\partial o_{p}^{(3)}(k)} \cdot f\left(n e t_{p}^{(3)}(k)\right)\left(1-f\left(n e t_{p}^{(3)}(k)\right) o_{i}^{(2)}(k)\right.
\end{aligned}
$$

Similarly, the learning algorithm of the hidden layer weight coefficient can be get:

$$
\Delta w_{i j}^{(2)}(k)=\alpha \Delta w_{i j}^{(2)}(k-1)-\eta o_{j}^{(1)}(k)\left[\left(1-f^{2}\left(n e t_{i}^{(2)}(k)\right) / 2\right] \sum_{p=1}^{3} \lambda_{p} w_{p i}^{(3)}(k)\right.
$$

Where,

$$
\lambda=e(k) \frac{\partial y(k)}{\partial \Delta u(k)} \frac{\partial \Delta u(k)}{\partial o_{p}^{(3)}(k)} f\left(n e t_{p}^{(3)}(k)\right)\left(1-f\left(n e t_{p}^{(3)}(k)\right)\right. \text {. }
$$

\section{EXPERIMENTAL STUDY}

According to the characteristics of the controlled object and the analysis of the learning algorithm, system learning rate $\eta=0.12$, inertia coefficient $\alpha=0.035$, the initial value of the weighted coefficient takes the random number[9] that based on the $-0.5 \sim 0.5$. Write the control program in MATLAB7.1, the program is applied to the experimental system, and its running results are shown in Figure V. In order to compare the control effect, the classical PID control results and fuzzy control results are given (In the same condition, the results of three kinds control method), as is shown in Figure VI and Figure VII.

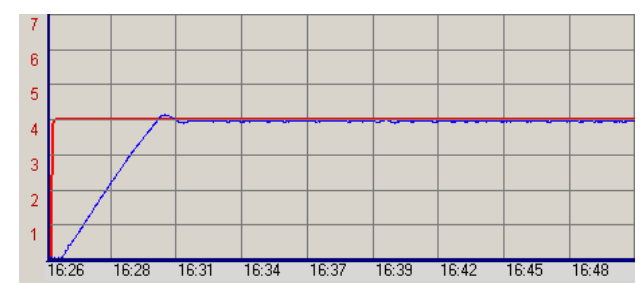

FIGURE V. OUTPUT CURVE OF THE NEURAL NETWORK PID CONTROL

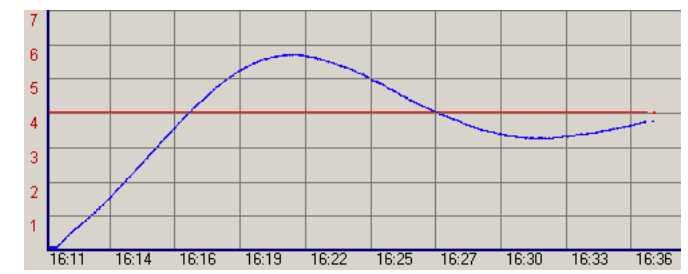

FIGURE VI. OUTPUT CURVE OF THE PID CONTROL

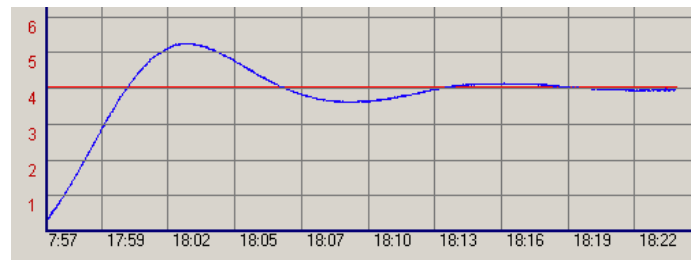

FIGURE VII. OUTPUT CURVE OF THE FUZZY CONTROL

From the results it can be conclude that the neural PID control improves the speediness and overshoot greatly, so its control effect is best, and the fuzzy control effect is better, and single PID control effect is poor.

\section{CONCLUSION}

In the view of control system of double-tank, the neural PID control is studied, the results are compared with the single PID control or single fuzzy control, the control effect is improved obviously. Although the control structure and algorithm are more complex, the experimental results show that it is a best control effect can be achieved by using the parameter adaptive neural PID control. 


\section{REFERENCES}

[1] Hong-xia NIU, Tao HOU. Research of fuzzy adaptive PID control on double-tank[J]. Industry Control and Applications, 2010, 29(12): 9-11.

[2] Tao HOU. Experimental research on neural network PID control based on hydraulic pressure of 2-container water tank [J]. Journal of Lanzhou Jiaotong University, 2009, 28(3): 41-43.

[3] Tao HOU, Hai-ying DONG. Design and experimental study of 2-container water tank fuzzy controller based on THJ-3[J]. Journal of Lanzhou Jiaotong University, 2005, 24(6): 107-109.

[4] Yu-li WEI. Fuzzy PID Control Study of Tank Liquid Level on Ultrasonic Sensor [J]. Automation and Instrumentation, 2016, 201(7): 9-11.

[5] Hong-xia NIU, Tao HOU. Experiment study of liquid level control based on ultrasonic sensor[J]. Industrial Instrumentation and Automation, 2016, 2016(4): 71-74.

[6] Zeng-qi SUN, Zai-xing ZHANG, Zhi-dong DENG. Intelligent control theory and technology[M]. tsinghua university press, 1997.

[7] Jie-qi ZHANG, Hong LIU. The application of neural network PID controller in the temperature control of superheated steam in power plant[J]. Computer Engineering and Applications, 2006, 30: 202-204.

[8] Jin-kun LIU. Advanced MATLAB control PID and simulation(The Second Edition)[M]. Beijing: Publishing House of Electronics Industry, 2004. 